\title{
FACTORES QUE INFLUENCIAN LA MOTIVACIÓN DE ESCOLARES POR LAS ÁREAS TECNOLÓGICAS E INGENIERÍA
}

\section{Antecedentes}

Durante los últimos 25 años, el porcentaje de mujeres matriculadas en carreras del área tecnológica de las universidades chilenas ha sido inferior al 20\%, traduciéndose en una baja representación femenina en carreras de ingeniería y, consecuentemente, en los sectores profesionales asociadas a estas carreras. Estos porcentajes representan los más bajos dentro de una gama de áreas de conocimientos, tales como administración y comercio, derecho, educación y salud.

Fundados en el reconocimiento de que hombres y mujeres poseen talentos equivalentes, los bajos porcentajes de participación femenina conllevan a que las empresas y la sociedad en general accedan a un menor número de profesionales capacitados en áreas tecnológicas e ingeniería, disminuyendo así la diversidad y productividad en la fuerza laboral (Williams \& Emerson, 2002).

Varias investigaciones se han centrado en estudiar las causas de esta tendencia. Algunas concluyen que los escolares se sienten más motivados por estudiar carreras en áreas tecnológicas e ingeniería cuando existe una buena experiencia en materias científicas, particularmente con matemáticas. Así, se ha hipotetizado que es más probable que escolares confiados en sus habilidades matemáticas, satisfechos con su desempeño e interesados en matemáticas estudien una carrera relacionada con ingeniería y/o tecnología (Eccles, 1987; Blaisdell, 1998).

Otros estudios señalan que la menor motivación manifestada por mujeres jóvenes hacia áreas tecnológicas e ingeniería se debería principalmente a la escasez de modelos femeninos (o mentors) en estas áreas, a la falta de motivación en las aulas y en el hogar y a los estereotipos hacia las carreras de ciencias e ingeniería (Esch, 2005). En 
Chile esta desmotivación se ve reflejada en los resultados de Prueba de Selección Universitaria (PSU) de matemáticas donde el puntaje promedio de las mujeres es inferior al de los hombres (Consejo de Rectores de las Universidades Chilenas, 2007). Esto se traduce en un número significativamente mayor de hombres postulando a carreras como ingeniería, en la cual matemáticas es la componente principal de la formación profesional.

\section{Factores asociados a la motivación por estudios superiores científico/matemáticos}

La motivación es un proceso sicológico multidimensional que entrega razón, orientación e intensidad de un comportamiento responsable principalmente por los resultados de un trabajo. Desde una perspectiva educacional, la motivación impulsa y orienta a los estudiantes a dedicarse a actividades académicas y determina el nivel de aprendizaje de estas actividades (Mwangi y McCaslin, 1994). Según Githua y Mwangi (2003), la motivación está compuesta por cuatro dimensiones: interés, relevancia, probabilidad de éxito y satisfacción en aprender matemáticas. Mientras el "interés" surge de la curiosidad del estudiante por aprender, responder y poner atención a una materia en particular, la "relevancia" corresponde al nivel de percepción que los estudiantes le dan al significado y valor del contenido de alguna materia en particular. La "probabilidad de éxito" indica la percepción de los escolares respecto de la posibilidad de resultar exitosos a través del control personal de sus comportamientos. Es decir, los alumnos se esforzarán en aprender cierta materia si creen que es probable obtener un buen desempeño. Finalmente, la "satisfacción" es el equilibrio sicológico de los alumnos debido a la experiencia de recompensas extrínsecas y la realización de crecimiento personal intrínseco (Githua y Mwangi, 2003).

En las últimas décadas, varios investigadores, particularmente de la Comunidad Europea y EE.UU., han centrado sus esfuerzos en determinar los principales factores que afectan la motivación de los escolares por proseguir estudios en áreas científicas o tecnológicas, principalmente matemáticas (Blaisdell, 1998; Bonnot \& Croizet, 2007; Eccles, 1987, 1989 y 1994; Esch, 2005; Farmer, 1987; Midgley et al., 
1989; Simpkins et al., 2006; Williams \& Emerson, 2002). En términos globales, estos estudios demuestran que los factores que influencian la motivación por las matemáticas son: autoestima, apoyo y expectativas de los padres, estatus socioeconómico, actitud de los profesores, experiencias pasadas, metas de corto y largo plazo, percepción del nivel de dificultad, estereotipos culturales, religión y desempeño académico. La literatura especializada lista una gran cantidad de variables que afectan directa e indirectamente la motivación de los escolares por las matemáticas. Sin embargo, pocos estudios han demostrado empíricamente la relación causal que los factores recién mencionados mantienen sobre esta motivación. Los estudios más referentes en este sentido son los desarrollados por Eccles (1994) y Farmer (1987) quienes, después de casi dos décadas de estudios sobre las habilidades de escolares por las matemáticas, proponen consistentes modelos conceptuales que describen los principales factores que influyen sobre las expectativas de éxito, desempeño futuro y decisiones vocacionales de los escolares. Ambos modelos concuerdan en que los factores autoconcepto, apoyo en el hogar y apoyo de los profesores serían los más influyentes sobre la motivación de los escolares hacia materias científicas y tecnológicas. El apoyo del hogar estaría estrechamente relacionado con las expectativas que los padres tienen sobre sus hijos, reflejado, por ejemplo, en la confianza expresada sobre las habilidades de los niños, en el orgullo y reconocimiento por el desempeño escolar alcanzado y en la comprensión de sus éxitos y frustraciones. Luego, sobre la base de estas expectativas, el apoyo hogareño influiría en el autoconcepto que los niños desarrollen sobre sus habilidades escolares.

Por su parte, el apoyo de los profesores está ligado a la empatía, amabilidad y trato equitativo que mantengan con sus estudiantes. Similar al apoyo proveniente del hogar, el apoyo del profesor dependerá de las expectativas y percepciones que éste tenga respecto de sus alumnos y alumnas.

\section{II.1. Autoconcepto por las matemáticas}

El autoconcepto está definido como la percepción del razonamiento, habilidades y capacidades personales por cierta materia en particular. Estudios empíricos han determinado que afecta la motivación de los 
escolares y por ende el rendimiento, esfuerzo y persistencia dedicada a las matemáticas. Por ejemplo, se afirma que las niñas poseen un autoconcepto inferior por las matemáticas que los niños, debido a la internalización de estereotipos producidos por presiones sociales que se traducen en un desempeño inferior en matemáticas (Bonnot y Croizet, 2007). Por lo tanto, las expectativas y creencias sobre hombres y mujeres son factores relevantes en el autoconcepto por las matemáticas.

\section{II.2. Apoyo de los profesores}

Estudios han demostrado una correlación positiva entre el nivel de apoyo que entregan los profesores en las aulas y la motivación (interés, relevancia y satisfacción) hacia diversas materias -específicamente por matemáticas y ciencias- de alumnos de la enseñanza media. Midgley et al. (1989) realizaron un estudio donde se determinó que la percepción de los alumnos respecto del apoyo de los profesores es un buen indicador de la calidad subjetiva de la relación profesor-alumno. La calidad de esta relación posee una asociación con la motivación académica de los alumnos y sus actitudes hacia sus colegios o liceos. Los autores concluyen que su diseño cuasi experimental sugiere que existe una relación causal entre la percepción del grado de apoyo de los profesores y el valor intrínseco por las matemáticas. Después de múltiples observaciones en las aulas, Eccles (1989) concluyó que los profesores inconscientemente tratan a sus alumnos y alumnas en forma diferente e inequitativa, específicamente respecto del uso de equipos computacionales y de laboratorio y la participación en clases. Además, observa que los profesores de matemáticas facilitan más oportunidades para aprender y practicar habilidades de liderazgo en sus clases a los hombres que a la mayoría de las mujeres. Los estudios de Midgley et al. (1989) y Eccles (1989) expresan que el apoyo que los profesores otorgan a sus estudiantes está estrechamente asociado con las expectativas y creencias que éstos mantienen sobre sus alumnos y alumnas. En consecuencia, dichas expectativas se reflejarán en un trato diferenciado al interior del aula con las consecuentes repercusiones sobre el autoconcepto que los alumnos desarrollan respecto de sus habilidades escolares. 


\section{II.3. Apoyo en el hogar}

Estudios sobre el impacto del ambiente familiar en la motivación y desempeño académico de los escolares han concluido que el apoyo de los padres es tan importante como la influencia que generan los establecimientos educacionales (Halawah, 2006; Cassidy y Lynn, 1991; Bonnot y Croizet, 2007). El factor "apoyo en el hogar" alude que los padres muestran ciertas expectativas y creencias de estereotipos de géneros en el desempeño y aptitudes académicas de sus hijos. Por ejemplo, Eccles (1989) indicó en su estudio que los padres afirmaron que sus hijas necesitaban dedicarles más tiempo a las matemáticas que sus hijos, ya que para ellas es una materia más difícil de comprender, aunque las habilidades académicas de ambos grupos son similares. Además, los padres afirmaron que la razón del éxito de sus hijos varones en las matemáticas es atribuida al talento natural por la asignatura, mientras que el buen desempeño de sus hijas en las clases de matemáticas es debido al esfuerzo. De esta manera, los padres desarrollan una mayor confianza en las aptitudes matemáticas de sus hijos que de sus hijas, generando un impacto directo sobre el autoconcepto y valores de los niños, lo que eventualmente incide en la elección de su carrera profesional.

\section{El estudio realizado y objetivos}

En la actualidad existe amplia evidencia teórica y empírica respecto de los factores que mayor influencia tienen sobre la motivación de escolares frente a sus opciones vocacionales. Sin embargo, la gran mayoría de estos estudios se ha efectuado en países desarrollados, los cuales poseen significativas diferencias socioeconómicas y culturales que impiden la extrapolación directa de resultados y conclusiones hacia sociedades menos desarrolladas. Lo anterior es particularmente efectivo en temas asociados con la educación primaria y secundaria.

El presente estudio pretende testear la hipótesis de que los factores autoconcepto, apoyo de los padres y apoyo de los profesores mantienen una relación causal directa sobre la motivación de los escolares frente a áreas tecnológicas e ingeniería (la figura 1 representa gráficamente la hipótesis planteada). 
Luego, el objetivo principal de este estudio es, a partir de la experiencia recopilada en países desarrollados durante las últimas décadas, determinar empíricamente la magnitud de la influencia que los factores apoyo en el hogar, apoyo de profesores y autoconcepto por las matemáticas generan sobre la motivación de escolares para estudiar carreras en áreas tecnológicas e ingeniería.

Figura 1. Modelo estructural en el cual los factores "apoyo en el hogar", "apoyo de profesores" y "autoconcepto por las matemáticas" mantienen una relación causal directa (o indirecta) sobre la motivación de los escolares por matemáticas.

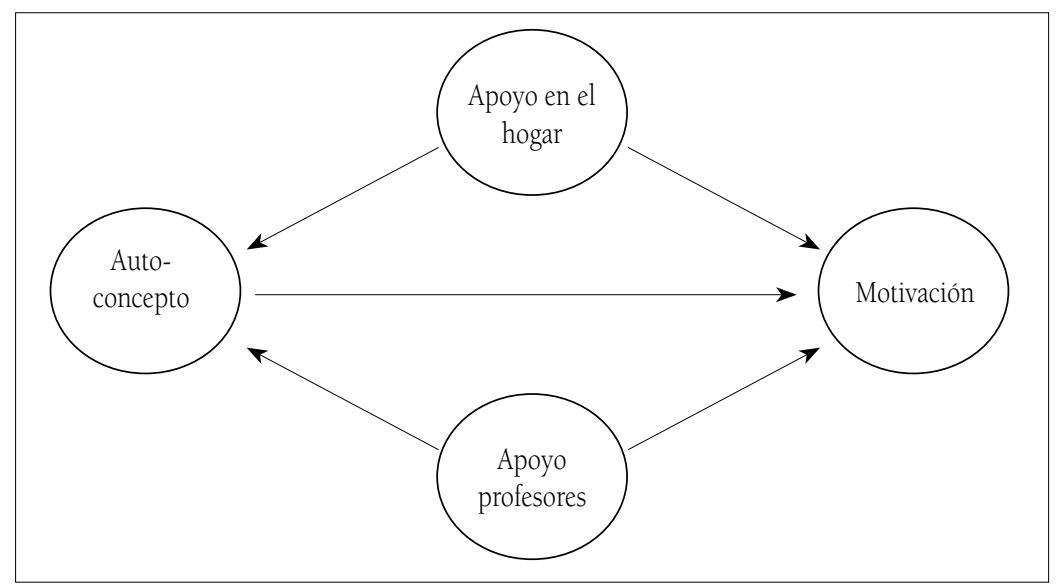

\section{Metodología}

\section{IV.1. Participantes}

Los datos del presente estudio fueron obtenidos de una encuesta implementada entre los meses de septiembre y octubre de 2009 en 33 establecimientos educacionales mixtos y no técnicos de 19 comunas de la ciudad de Santiago. Estos colegios fueron convenientemente seleccionados en función de la disponibilidad directiva de participar en el estudio y de su localización geográfica, se encuentran uniformemente distribuidos en la ciudad y constituyen una muestra representativa del total de establecimientos municipales, particulares subvencionados y particulares pagados de la ciudad de Santiago. Un total de 1.697 escolares (55,7\% niñas y promedio de edad de 15,9 años) completaron anónimamente la encuesta. 


\section{2 Diseño de la encuesta}

Con el propósito de identificar los principales elementos que pueden influenciar la motivación de escolares hacia áreas tecnológicas e ingeniería, se realizó un grupo focal conformado por ocho expertos (sicólogos, sociólogos, pedagogos, entre otros) con intereses comunes por las áreas de educación, género e ingeniería y tecnología. Las variables identificadas por este panel experto fueron, en general, consistentes con aquellas representadas en los modelos causales teorizados por Eccles (1994) y Farmer (1987), y descritas en la Sección II. Luego de seleccionados los factores que serían medidos, la encuesta fue diseñada y posteriormente validada mediante la realización de un grupo focal con ocho estudiantes de enseñanza media de la ciudad de Santiago.

Un total de 34 variables (ver anexo I) fueron utilizadas para medir los cuatro factores del modelo causal mostrado en la figura 1. El primer factor, "apoyo en el hogar", fue medido por cinco variables ( $\alpha$ de Cronbach $=0,79)$ adaptadas del estudio de Halawah (2006). Cada variable fue cuantificada utilizando una escala Likert 5-puntos desde "Nunca (1)" hasta "Siempre (5)". El factor "apoyo de los profesores" fue medido por medio de tres variables ( $\alpha$ de Cronbach $=$ 0,71) adaptadas del estudio de Midgley et al. (1989). Para cuantificar estas variables se utilizó la misma escala Likert de las variables del primer factor. El tercer factor, "autoconcepto por las matemáticas", se midió a través de seis variables ( $\alpha$ de Cronbach $=0,91$ ) adaptadas del estudio realizado por Simpkins et al. (2006). Para cuantificar estas variables se utilizaron tres escalas Likert 5-puntos con distintos rótulos extremos: por ejemplo, la pregunta "¿cómo eres para las matemáticas?" se midió desde "Muy malo (1)" a "Muy bueno (5)", y la pregunta "en comparación con tus otras asignaturas ¿cómo te va en matemáticas?" se midió desde "Mucho peor (1)" a "Mucho mejor (5)". Finalmente, el factor "motivación" fue medido en cuatro dimensiones (interés, relevancia, satisfacción y probabilidad de éxito) por medio de un total de 20 variables ajustadas del estudio realizado por Githua y Mwangi (2003): seis variables fueron cuantificadas para medir "interés" ( $\alpha$ de Cronbach $=0,92)$, cinco para "relevancia" ( $\alpha$ de Cronbach $=0,83)$, seis para "probabilidad de éxito" ( $\alpha$ de Cronbach $=0,84$ ) y tres para 
"satisfacción" ( $\alpha$ de Cronbach $=0,87$ ). Para cada una de estas variables se utilizó una escala Likert 5 -puntos desde "Totalmente en desacuerdo (1)" hasta "Totalmente de acuerdo (5)".

Para mayor detalle de las variables utilizadas referirse al anexo I.

\section{Análisis de datos}

Para testear el modelo causal postulado se utilizaron procedimientos de modelación de ecuaciones estructurales. Los parámetros del modelo fueron estimados por medio del programa AMOS 5.0 de SPSS. Para evaluar el ajuste del modelo se emplearon dos índices: Comparative Fit Index (CFI) y Root Mean Square Error of Approximation (RMSEA). El CFI toma valores entre 0 y 1 , donde un mayor valor indica un mejor ajuste del modelo. Tradicionalmente, se considera que un modelo posee un buen ajuste cuando el CFI es superior a 0,90. Por su parte, el índice RMSEA sugiere un buen ajuste del modelo cuando posee valores no superiores a 0,05. Para testear la hipótesis nula de que el indicador RMSEA no es superior a 0,05, se utiliza el test PCLOSE. Un valor $-p$ de este test debería ser superior a 0,5 para rechazar la hipótesis nula.

Para evaluar la existencia de un mal ajuste del modelo se utilizó el Índice de Modificación (IM). El valor IM de un parámetro indica que, si el parámetro fuera estimado libremente (sin fijarle un valor) en un modelo subsecuente, la magnitud del estadístico $\chi^{2}$ global disminuiría a lo menos en esa cantidad. Altos valores en el IM de un parámetro sugieren la presencia de covarianza entre errores o de una variable con cargas multifactoriales.

Los análisis fueron realizados en tres etapas. Primero, los datos observados fueron ajustados al modelo hipotetizado. Fueron testeados cuatro modelos, uno para cada dimensión del factor "motivación"; es decir, un modelo para "interés por las matemáticas", otro para "relevancia de las matemáticas", otro para "satisfacción" y un último modelo para "probabilidad de éxito". En la segunda etapa fue testeado el modelo en sus cuatro versiones (uno para cada dimensión de "motivación"), tanto para hombres como para mujeres. Finalmente, 
en la tercera etapa se exploraron las diferencias de género para cada factor incluidas en los modelos causales.

\section{Resultados}

La figura 2 muestra el modelo final resultante para la dimensión "interés en matemáticas" del factor motivación. Si bien el modelo hipotetizado en la figura 1 generó un buen ajuste de los datos (CFI = 0,951 y RMSEA $=0,057)$, el test PCLOSE tomó un valor radicalmente bajo $(\mathrm{PCLOSE}=0,000)$. Al agregar tres correlaciones entre errores sugeridas por los valores IM se obtuvo un mejor ajuste del modelo $(\mathrm{CFI}=0,967, \mathrm{RMSEA}=0,048$ y PCLOSE $=0,829)$. La figura 2 muestra que los datos claramente soportan la hipótesis del modelo causal hipotetizado para la dimensión "interés por las matemáticas". Las estimaciones de cada arco representan coeficientes estandarizados.

Los resultados indican que el factor "autoconcepto en matemáticas" es la variable que mayor influencia tiene sobre el "interés en matemáticas" de los escolares. Si bien el factor "apoyo de profesores" también mantuvo una relación causal directa y significativa con "interés por matemáticas", su efecto directo fue aproximadamente ocho veces menor que el asociado al factor "autoconcepto en matemáticas". Sorpresivamente, el factor "apoyo en el hogar" no evidenció una relación estadísticamente significativa $(p<0,001)$ con "interés por las matemáticas", manteniendo sólo una débil relación con "autoconcepto en matemáticas". 
Figura 2. Modelo final de la dimensión "interés por las matemáticas" del factor "motivación". Los valores representan estimaciones estandarizadas, $N=1.697$. Todos los coeficientes son significativos $(p<0,001)$, con excepción del coeficiente 0,02 ponderando a la variable "apoyo en el hogar" para explicar la variable dependiente "interés".

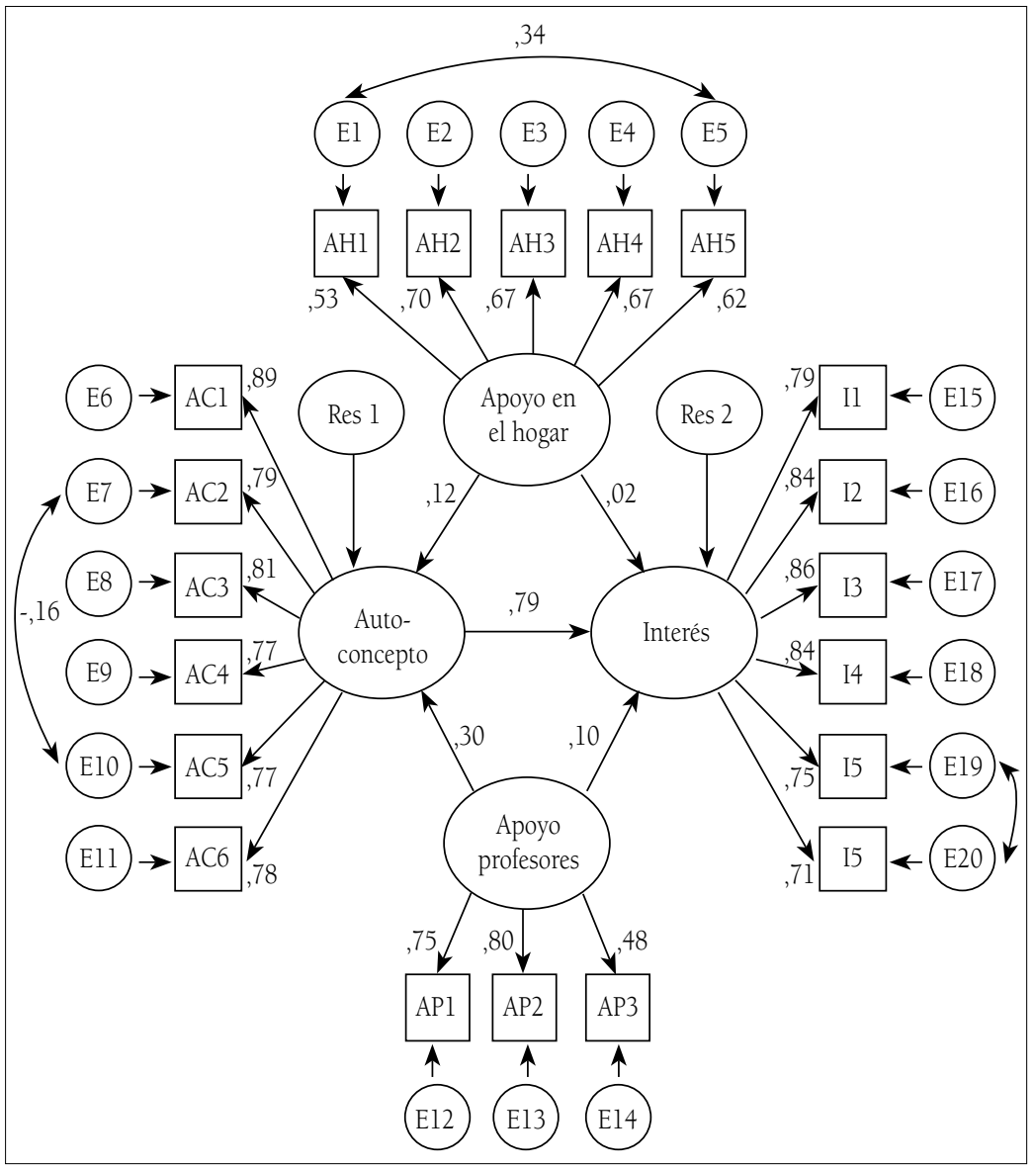

Cuando el modelo causal de la figura 1 fue testeado para la dimensión "relevancia" del factor "motivación", los resultados fueron similares a los entregados en la figura 2 para "interés en matemáticas". El modelo hipotetizado logró un buen ajuste de los datos (CFI = 0,949 y RMSEA $=0,053$ ), aunque el test PCLOSE tuvo un valor significativamente bajo (PCLOSE $=0,048$ ). Sobre la base del valor del IM asociado a cada parámetro se determinó la existencia de dos pares de variables de error correlacionadas. El modelo final es mostrado en 
la figura $3(\mathrm{CFI}=0,960$, RMSEA $=0,048$ y PCLOSE $=0,872)$. Al igual que en el modelo de la figura 2, la influencia directa que el factor "apoyo en el hogar" genera sobre la dimensión "relevancia" no fue estadísticamente significativa. Como se presenta en la figura 3, los factores que mayor impacto causan sobre la dimensión "relevancia" son "apoyo de los profesores" y "autoconcepto en matemáticas", siendo el impacto directo de este último más de tres veces superior al del primero.

Figura 3. Modelo final de la dimensión "relevancia de las matemáticas" del factor "motivación". Los valores representan estimaciones estandarizadas. Todos los coeficientes son significativos $(p<0,001)$, con excepción del coeficiente 0,07 ponderando a la variable "apoyo en el hogar" para explicar la variable dependiente "interés".

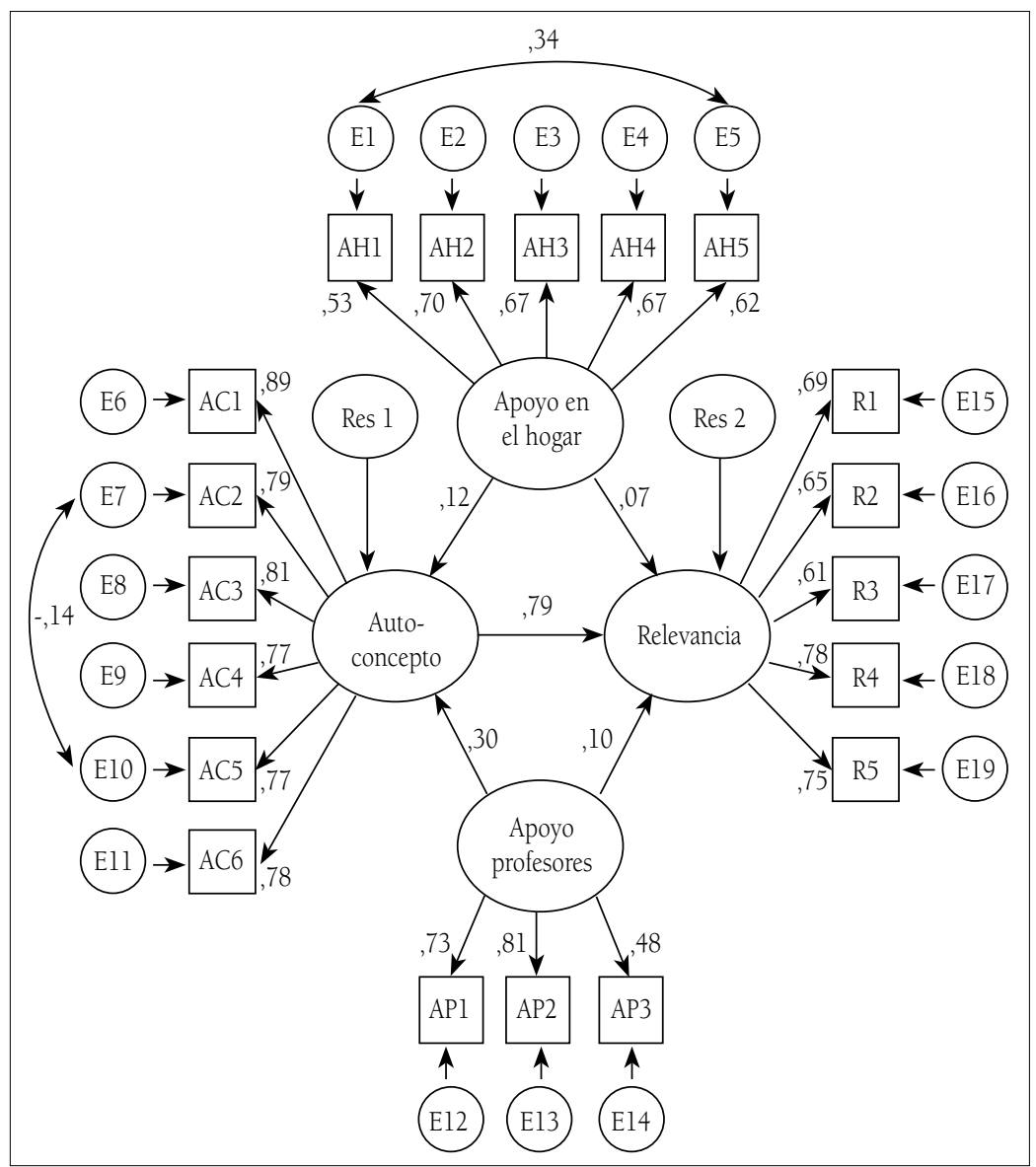


El modelo causal resultante para la dimensión "satisfacción" del factor "motivación" es mostrado en la figura 4. Si bien el modelo hipotetizado en la figura 1 generó un buen ajuste de los datos (CFI = 0,949 y RMSEA $=0,061$ ), el test PCLOSE tomó un valor significativamente bajo (PCLOSE $=0,000$ ). Sobre la base del valor del IM asociado a cada parámetro se determinó la existencia de tres pares de variables de error correlacionadas. Si bien el modelo final de la figura 4 (CFI = 0,967, RMSEA $=0,049$ y PCLOSE $=0,587$ ) mantiene una tendencia similar a los modelos obtenidos para las dimensiones "interés" y "relevancia", en esta ocasión el factor "apoyo de los profesores" fue el que mayor influencia directa mantiene sobre la dimensión "satisfacción". Nuevamente, el factor "apoyo en el hogar" no fue significativo.

Figura 4. Modelo final de la dimensión "satisfacción" del factor "motivación". Los valores representan estimaciones estandarizadas. Todos los coeficientes son significativos $(p<0,001)$, con excepción del coeficiente 0,04 ponderando a la variable "apoyo en el hogar" para explicar la variable dependiente "interés".

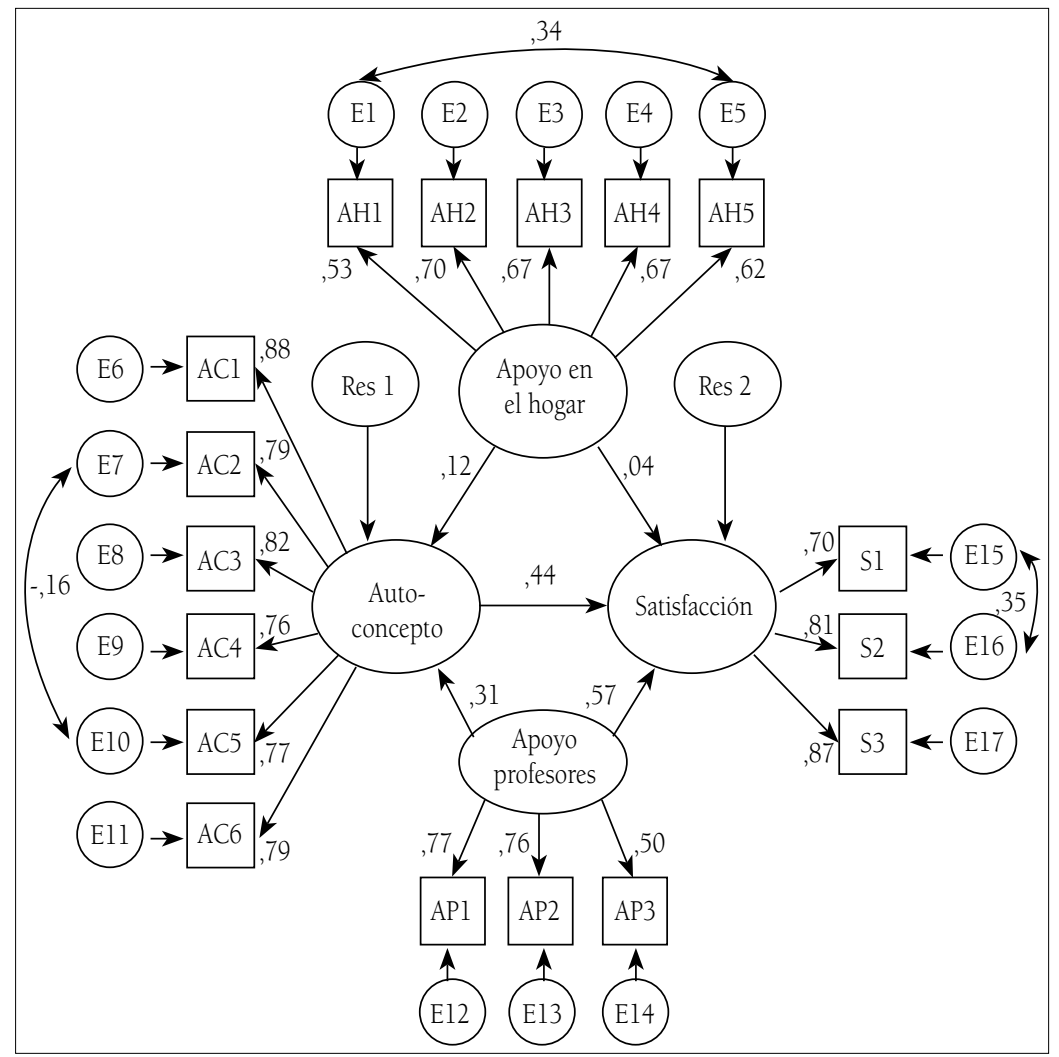


Finalmente, el modelo mostrado en la figura 5 representa las relaciones causales que los factores "apoyo en el hogar", "autoconcepto" y "apoyo de los profesores" causan sobre el factor "probabilidad de éxito". Al igual que en los modelos anterior, los valores del IM indicaban la existencia de tres pares de variables de error correlacionadas. El modelo final tuvo un muy buen ajuste ( $\mathrm{CFI}=$ 0,960, RMSEA $=0,047$ y PCLOSE $=0,928$ ). A diferencia de lo ocurrido con las otras tres dimensiones asociadas con el factor "motivación por las matemáticas", todos los factores del modelo generaron un efecto directo estadísticamente significativo sobre la dimensión "probabilidad de éxito", donde los factores "apoyo en el hogar" y "apoyo de los profesores" mantuvieron un similar efecto, mientras que el factor "autoconcepto" mantuvo un efecto sobre "probabilidad de éxito" cerca de seis veces superior al de los otros dos factores.

Figura 5. Modelo final de la dimensión "probabilidad de éxito" del factor "motivación". Los valores representan estimaciones estandarizadas. Todos los coeficientes son significativos $(p<0,001)$.

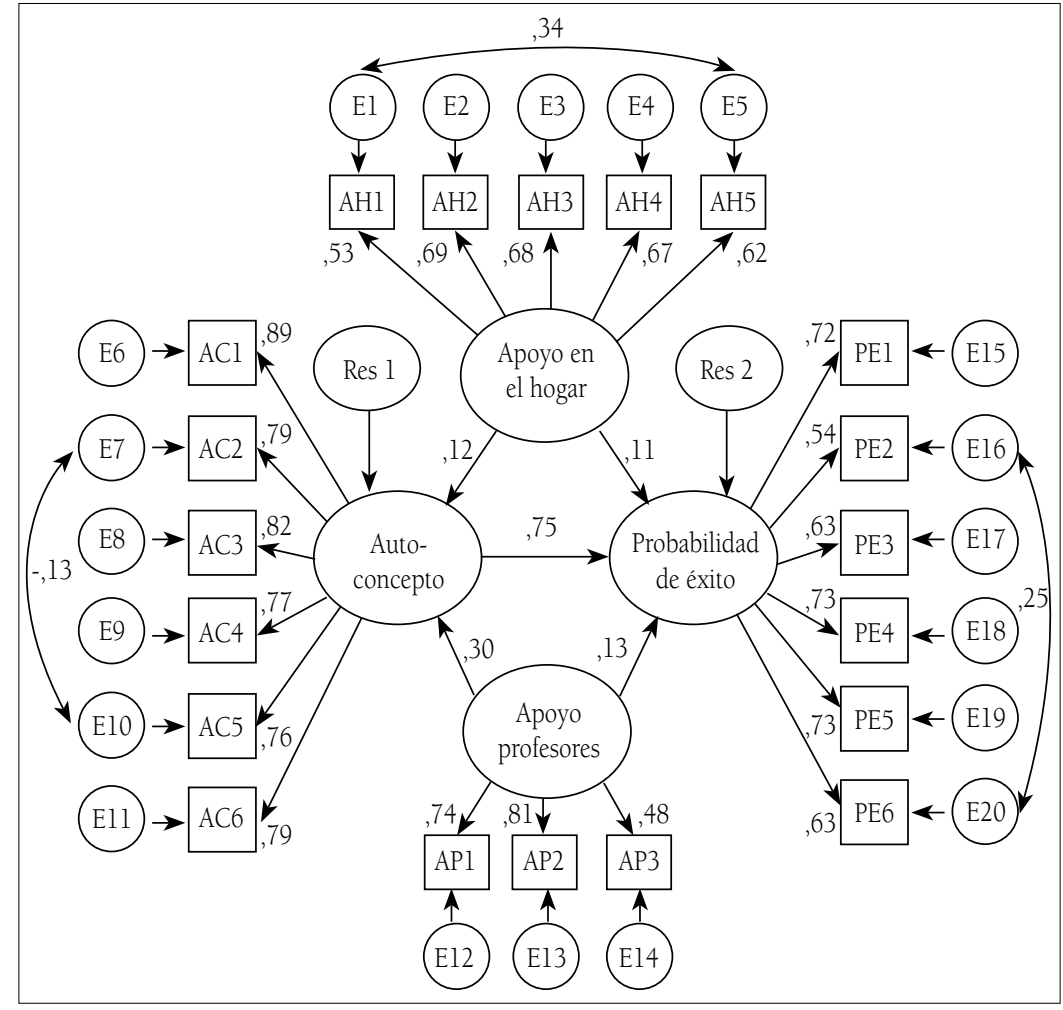


Cada uno de los cuatro modelos descritos fue analizado individualmente para explorar las posibles diferencias según género. Si bien todos los modelos causales resultantes se ajustaron adecuadamente a los datos (según índices CFI y RMSEA), no existieron diferencias estadísticamente significativas.

Finalmente, las diferencias respecto de género fueron exploradas para cada factor incluido en el modelo. Los resultados indican que sólo para los factores "apoyo en el hogar" y "autoconcepto" existió una diferencia estadísticamente significativa $(p<0,05)$. Mientras las mujeres percibieron un mayor apoyo en el hogar, los hombres manifestaron poseer mayores habilidades en matemáticas que las mujeres.

\section{Discusión y conclusiones}

En la literatura especializada existe amplio consenso respecto de los factores que mayor influencia tienen sobre la motivación de escolares frente de sus opciones vocacionales. Sin embargo, un escaso número de estudios han sido efectuados en países latinoamericanos. El objetivo principal del presente estudio fue, a partir de la experiencia recopilada en países desarrollados durante las últimas décadas, determinar empíricamente la magnitud de la influencia que los factores "apoyo en el hogar", "apoyo de profesores" y "autoconcepto por las matemáticas" generan sobre la motivación de escolares para estudiar carreras en áreas tecnológicas e ingeniería.

Los resultados de este estudio sugieren que el factor "autoconcepto" es la variable que posee la mayor relación causal sobre las distintas dimensiones motivacionales de los escolares (interés, probabilidad de éxito, satisfacción y relevancia). Al igual que lo reportado en estudios previos (Bonnot \& Croizet, 2007; Eccles, 1987, 1989 y 1994), las niñas poseen un autoconcepto inferior por las matemáticas que los niños. Luego, a partir de este bajo autoconcepto de habilidades matemáticas declarada por las niñas (relativo a la opinión de los niños), podría explicarse la menor motivación de las mujeres jóvenes por las áreas tecnológicas e ingeniería. En consecuencia, cualquier estrategia comunicacional y educacional 
centrada en reforzar el autoconcepto de habilidades matemáticas de las niñas resultaría en un incremento motivacional frente a estas áreas. Huyer (2005) sugiere fomentar el cambio en la percepción de los profesores en las capacidades y habilidades de las niñas por las matemáticas, eliminar estereotipos de género, incrementar la participación equitativa en las aulas y utilizar imágenes, lenguaje y modelos tanto masculinos como femeninos en los textos escolares y los medios relacionados con ciencias y matemáticas.

El factor "autoconcepto" es directa y significativamente influenciado por el "apoyo en el hogar" y el "apoyo de los profesores". Estos resultados sugieren que tanto los padres como los profesores deberían invertir mayor tiempo y esfuerzo en desarrollar las percepciones personales de autoconcepto por las matemáticas. Parte del nivel del autoconcepto de los escolares está dado por expectativas y percepciones de sus logros académicos proporcionados por sus referentes significativos, especialmente padres y profesores. De esta manera, si los padres y profesores muestran expectativas y percepciones positivas hacia las capacidades de los escolares, entonces es probable que éstos obtenga un mejor desempeño académico y, en consecuencia, presenten altos niveles de autoconcepto por sus habilidades (Simpkins et al., 2006).

Luego, medidas educacionales destinadas a reforzar la labor motivacional de profesores y padres impactarán positivamente sobre el interés, relevancia, satisfacción y probabilidad de éxito de los escolares frente a las matemáticas. Como resultado de estas medidas, que incluyen la distribución de videos y herramientas educacionales o capacitaciones pedagógicas, se debería aumentar la conciencia acerca de los sesgos de género que existen hacia las áreas de ingeniería y tecnología. De este modo, los profesores debieran enseñar matemáticas con un diseño curricular diferente al actual, con contenidos más atractivos hacia las niñas, tales como economía, ecología e inquietudes sociales.

Varios estudios han declarado que el "apoyo en el hogar" es tanto o más relevante que el apoyo que se da en las aulas (Eccles, 1994; Farmer, 1987; Githua \& Mwangi, 2003; Halawah, 2006; Cassidy \& 
Lynn, 1991; Bonnot \& Croizet, 2007). Sin embargo, los resultados del presente estudio contradicen esta afirmación. Mientras el factor "apoyo de los profesores" mantuvo un efecto significativo sobre las dimensiones de "motivación por las matemáticas", el factor "apoyo en el hogar" sólo fue levemente significativo para una dimensión de motivación (probabilidad de éxito). Este resultado implica que, dado el contexto actual de la educación nacional, la labor motivacional de los profesores sería más influyente que la entregada en el hogar. Es decir, los pensamientos y acciones de los profesores pesan de manera importante por sobre los de los padres en el autoconcepto de los alumnos y, por ende, en la motivación de los escolares por escoger una carrera científica/matemática. El apoyo de los profesores, dado por las expectativas y percepciones que éstos tengan respecto de sus alumnos y alumnas, está ligado a la empatía, amabilidad y trato equitativo en las salas de clases. Luego, los profesores de matemáticas poseen una posición privilegiada dentro del sistema educacional para motivar y despertar la participación y el interés por las matemáticas, especialmente el de las niñas. Al aumentar el nivel de todas las dimensiones de la motivación se facilitará el aprendizaje, la diversión y la retención de la materia enseñada (Githua \& Mwangi, 2003).

\section{Referencias bibliográficas}

Blaisdell, S. (1998) Predictors of Women's Entry into Engineering: Why Academic Preparation is not Sufficient. Proceedings of Frontiers in Education Conference, 1, pp. 221-225.

Bonnot, V. \& Croizet, J-C. (2007) Stereotype internalization, math perceptions, and occupational choices of women with counter-stereotypical university majors. Swiss Journal of Psychology, 66(3), pp. 169-178.

Cassidy, T. \& Lynn, L. (1991) Achievement motivation, educational attainment, cycles of disadvantage and social competence: Some longitudinal data. British Journal of Educational Psychology, 61(1), pp. 1-12.

Consejo Superior de Educación, CSE (2007) Indices 2008, matrícula total pregrado por tipo de institución y área de conocimiento. Santiago de Chile: CSE.

Eccles, J. S. (1987) Gender roles and women`s achievement-related decisions. Psychology of Women Quarterly, 11, pp. 135-172.

Eccles, J. S. (1989) Bringing Young Women to Math and Science. En Crawford, 
M \& Gentry M. (eds.) Gender and Thought. New York: Spring-Verlag, pp. 36-58.

Eccles, J. S. (1994) Understanding women's educational and occupational choices. Psychology of Women Quarterly, 18, pp. 585-609.

Esch, M. (2005) Career building for qualified young women managers and leaders in science and engineering: A public-private partnership initiative between technical universities and companies. Proceedings of the International Symposium on Women and ICT: Creating Global Transformation, 126. Baltimore, MD.

Farmer, H. S. (1987) A multivariate model for explaining gender differences in career and achievement motivation. Educational Researcher, 16(2), pp. 5-9.

Githua, B. N. \& Mwangi, J. G. (2003) Students mathematics self-concept and motivation to learn mathematics: relationship and gender differences among Kenya's secondary-school students in Nairobi and Rift Valley provinces. International Journal of Educational Development, 23, pp. 487-499.

Halawah, I. (2006) The effect of motivation, family environment, and student characteristics on academic achievement. Journal of instructional psychology, 33 (2), pp. 91-99.

Huyer, S. (2005) New technologies creating new opportunities for women. An international perspective. Women in Global Science and Technology (WIGSAT), 49th Session of the Commission on the Status of Women, New York.

Midgley, C.; Feldlaufer, H. \& Eccles, J. S. (1989) Student/teacher relations and attitudes toward mathematics before and after the transition to junior high school. Child Development, 60(4), pp. 981-992.

Mwangi, J. \& McCaslin, N. (1994) The motivation of Kenya's Rift Valley extension agents. Journal of Agricultural Education, 35(3), pp. 35-43.

Simpkins, S.D.; Davis-Kean, P.E. \& Eccles, J.S. (2006) Math and science motivation: A longitudinal examination of the links between choices and beliefs. Developmental Psychology, 42(1), pp. 70-83.

Williams, F.M. \& Emerson; C.J. (2002) Becoming Leaders: A Handbook for Women in Science, Engineering and Technology (3 $3^{\text {rd }}$ edition). St. John's, NL: Canada Memorial University of Newfoundland.

Recibido: 27 de octubre de 2009

Aceptado: 5 de noviembre de 2009 


\section{ANEXO I}

Cuestionario sobre el apoyo en el hogar

AH1. En mi hogar me reconocen cuando me saco buenas notas.

AH2. En mi hogar entienden mis sentimientos.

AH3. En mi hogar confían en mis habilidades.

AH4. En mi hogar les gusta hacer cosas conmigo.

AH5. En mi hogar se sienten orgullosos cuando me saco buenas notas.

Cuestionario sobre el apoyo de los profesores

AP1. Al profesor(a) de matemáticas le importa cómo se sienten en clase sus alumnos y alumnas.

AP2. El profesor(a) de matemáticas es amable con la mayoría de los alumnos y alumnas del curso.

AP3. El profesor(a) de matemáticas corrige las tareas o pruebas a todos por igual.

Cuestionario sobre el autoconcepto

AC1. ¿Cómo eres para las matemáticas?

AC2. ¿Cómo eres tú para aprender algo nuevo en matemáticas?

AC3. ¿Cómo crees que te irá este año en matemáticas?

AC4. ¿Cómo crees que te iría en una carrera que requiere de habilidades matemáticas?

AC5. En comparación con tus otras asignaturas, ¿cómo te va en matemáticas?

AC6. Si tuvieras que hacer un ranking respecto de las notas de matemáticas de tus compañeros y compañeras, ¿en qué lugar te ubicarías tú?

Cuestionario sobre la motivación: satisfacción

S1. Estoy de acuerdo con la forma en que se enseña matemáticas en mi colegio/liceo.

S2. Estoy satisfecho(a) con la forma que aprendo matemáticas en mi colegio/ liceo.

S3. Me siento cómodo(a) en clases de matemáticas.

Cuestionario sobre la motivación: interés

I1. ¿Cuánto te gusta hacer ejercicios de matemáticas?

I2. Me gusta aprender matemáticas.

I3. Disfruto el tiempo que le dedico a las matemáticas.

I4. Estoy muy motivado(a) en aprender matemáticas.

I5. En general, encuentro que las matemáticas son muy interesantes.

I6. En comparación con mis otras actividades escolares, me gustan mucho más las matemáticas. 
Cuestionario sobre la motivación: probabilidad de éxito

PEl. A veces resuelvo exitosamente las actividades de matemáticas entregadas en clase.

PE2. Aprender matemáticas me da la oportunidad de desarrollarme en el futuro.

PE3. Espero que me vaya bien en matemáticas.

PE4. Soy capaz de trabajar solo(a) en ejercicios de matemáticas.

PE5. Espero resolver cualquier problema de matemáticas si son de mi nivel educacional.

PE6. Espero ser capaz de aplicar fácilmente las matemáticas en cualquier situación de mi vida cotidiana.

Cuestionario sobre la motivación: relevancia

R1. ¿Cuánto crees que te servirá lo que aprendes en matemáticas para cuando trabajes?

R2. Encuentro que son importantes las actividades desarrolladas en clases de matemáticas.

R3. El tema de las matemáticas está vinculado a mis experiencias diarias.

R4. Las matemáticas son importantes para mis necesidades y metas futuras.

R5. Para mí es muy importante ser bueno en matemáticas. 